\title{
Cancer in ANCA-Associated Glomerulonephritis: A Registry-Based Cohort Study
}

\author{
Sanjeevan Sriskandarajah, ${ }^{1}$ Leif Bostad, ${ }^{1,2}$ Tor Åge Myklebust, ${ }^{3}$ Bjørn Møller, ${ }^{3}$ \\ Steinar Skrede, ${ }^{4,5}$ and Rune Bjørneklett ${ }^{1,6}$ \\ ${ }^{1}$ Department of Clinical Medicine, University of Bergen, Bergen, Norway \\ ${ }^{2}$ Department of Pathology, Haukeland University Hospital, Bergen, Norway \\ ${ }^{3}$ Department of Clinical and Registry-Based Research, Cancer Registry of Norway, Institute of Population-Based Cancer Research, \\ Oslo, Norway \\ ${ }^{4}$ Department of Clinical Science, University of Bergen, Bergen, Norway \\ ${ }^{5}$ Department of Medicine, Haukeland University Hospital, Bergen, Norway \\ ${ }^{6}$ Emergency Care Clinic, Haukeland University Hospital, Bergen, Norway
}

Correspondence should be addressed to Sanjeevan Sriskandarajah; sanjeevan87@hotmail.com

Received 22 September 2017; Accepted 27 November 2017; Published 18 December 2017

Academic Editor: Tibor Nadasdy

Copyright (C) 2017 Sanjeevan Sriskandarajah et al. This is an open access article distributed under the Creative Commons Attribution License, which permits unrestricted use, distribution, and reproduction in any medium, provided the original work is properly cited.

Background. Immunosuppressive therapy for antineutrophil cytoplasmic antibody-associated vasculitis has been associated with increased malignancy risk. Objectives. To quantify the cancer risk associated with contemporary cyclophosphamide-sparing protocols. Methods. Patients from the Norwegian Kidney Biopsy Registry between 1988 and 2012 who had biopsy-verified pauciimmune glomerulonephritis and positive antineutrophil cytoplasmic antibody (ANCA) serology were included. Standardised incidence ratios (SIRs) were calculated to compare the study cohort with the general population. Results. The study cohort included 419 patients. During 3010 person-years, cancer developed in 41 patients (9.79\%); the expected number of cancer cases was 37.5 (8.95\%). The cohort had SIRs as follows: 1.09, all cancer types (95\% CI, 0.81 to 1.49); 0.96, all types except nonmelanoma skin cancer (95\% CI, 0.69 to 1.34 ); 3.40, nonmelanoma skin cancer (95\% CI, 1.62 to 7.14 ); 3.52, hematologic cancer (95\% CI, 1.32 to 9.37); 2.12, posttransplant cancer ( $95 \%$ CI, 1.01 to 4.44 ); and 1.53, during the $1-5$-year follow-up after diagnosis (95\% CI, 1.01 to 2.32). Conclusions. Cancer risk did not increase significantly in this cohort with ANCA-associated glomerulonephritis. However, increased risk of nonmelanoma skin cancer, posttransplant cancer, and hematologic cancer indicates an association between immunosuppression and malignancy.

\section{Introduction}

Historically, antineutrophil cytoplasmic antibody- (ANCA-) associated vasculitis (AAV) was a fatal disease. The introduction of cyclophosphamide (CYC) treatment in the 1960s improved the prognosis and made long-term survival possible for patients with AAV $[1,2]$. However, evidence soon emerged that the long-term survival of patients with AAV was associated with significant morbidity including a substantially increased cancer risk [3-7]. Immunosuppressive therapy using CYC was particularly associated with malignancy. Although the severe adverse effects have elicited a search for less toxic treatment regimens, CYC still remains the first-line drug [8].

High occurrence of cancer has been demonstrated in patients with AAV treated with cumulative CYC doses exceeding $36 \mathrm{~g}$ [9-11] or when the treatment duration lasted for more than a year $[4,12-14]$. In the current clinical practice, cumulative CYC doses and treatment duration rarely exceed these limits [15-18], which questions the relevance of previous CYC-associated increased cancer risk observations in patients with AAV. There is a relative paucity of data regarding cancer risk in AAV patients treated with current therapy protocols, and, to our knowledge, only 4 studies have published 
such data $[5,11,13,19]$. In these studies, increased incidence of malignancy was observed. After excluding nonmelanoma skin cancer (NMSC) from the analyses, the incidence of malignancy no longer significantly increased in any of these investigations. However, these 4 studies were relatively small, with a limited statistical power to detect small- to mediumrange associations between cancer and AAV. Furthermore, most of these reports are single-center studies, which limits their generalizability.

Interestingly, in the most recent study, the standardised incidence ratio (SIR) of all cancer types treated with CYC was 4.61 (95\% confidence interval (CI) 1.16 to 39.38 ) times higher than that with rituximab-based therapy. However, after excluding NMSC from their analysis, the risk was only 1.30 (95\% CI not reported) times higher with CYC compared with rituximab-based therapy [19].

To further investigate the association of cancer and AAV, we analysed data from the Norwegian Kidney Biopsy Registry (NKBR) and the Norwegian Cancer Registry. Additionally, we merged data from the present and the 4 recent studies on this topic and investigated the cancer risk in a total of 1532 patients with AAV diagnosed after 1988.

\section{Materials and Methods}

The study was approved by the Regional Committees for Medical and Health Research Ethics (REC South-East 2013/ 1083).

2.1. Study Population and Registries. The NKBR was established in 1988. We estimate that $\sim 90 \%$ of all kidney biopsies are registered. The registry contains morphological, laboratory, and clinical data collected when the biopsy was performed. The Norwegian Cancer Registry was established in 1953. Reporting to this registry is mandatory and based on reports from clinical and pathological departments and death certificates. A near complete registration (98-99\%) of solid tumors, except for basal skin cell carcinomas, is documented [20]. Annual sex-specific incidence rates for cancer and cancer subtypes are available for age groups and time periods in 5-year intervals. These data allow for an accurate calculation of the expected cancer case numbers in the study cohort. The Norwegian Cause of Death Registry is part of Statistics Norway and based on the mandatory Norwegian death certificate. The Norwegian Renal Registry was established in 1980 and has registered all patients with end-stage renal disease, defined by the commencement of maintenance dialysis or receiving kidney transplantation.

2.2. Data Collection and Definitions. We included patients registered in the NKBR and diagnosed from 1988 to 2012, with a pauci-immune necrotising glomerulonephritis and a positive ANCA serology. Patients with cancer prior to the AAV diagnosis were excluded. Baseline clinical data, including sex, age, ANCA specificity, and estimated glomerular filtration rate (eGFR) (determined by the Modification of Diet in Renal Disease equation) [21] were obtained from the NKBR. The primary study end-point was incidence of cancer. By using the unique 11-digit Norwegian personal number, the study cohort was linked with the Norwegian Cancer Registry to identify the cancer incidence. Causes of deaths in the study cohort were identified through linkage with the Norwegian Cause of Death Registry and classified as vascular, malignant, infectious, or active inflammation and other causes. The observation period was from the kidney biopsy date to incident cancer, end of 2013, or death, whichever came first. Patients with end-stage renal disease and those receiving kidney transplants were identified through record linkage with the Norwegian Renal Registry. When we calculated the cancer risk in transplanted patients, the observation period started from the kidney transplantation date.

2.3. SIR Calculation. We calculated the SIR as the ratio between the observed and expected cancer case numbers in the cohort. The expected cancer case number was calculated as follows: first, the number of person-years was calculated in the cohort, stratified by 5 -year age groups and 1-year time periods. Second, this person-time was multiplied with the corresponding incidence rate in the general population to get the expected number of cases in each age group and 1-year period. The total number of expected cancer cases was then calculated as the sum of expected cases across age groups and time periods. The observed cancer case number was determined by record linkage of the study cohort and the Norwegian Cancer Registry using the 11-digit unique Norwegian personal number. A Poisson distribution of cancer incidence was assumed when 95\% CIs were calculated. We calculated the SIR throughout the study period and stratified in accordance with disease duration periods $(0-1,1-5,5-10$, and $>10$ years after kidney biopsy), sex, ANCA specificity [cytoplasmic ANCA (C-ANCA)/proteinase 3 ANCA (PR3-ANCA) or perinuclear ANCA (P-ANCA)/myeloperoxidase ANCA (MPO-ANCA)], time periods (1988-2002 and 2003-2012), and posttransplantation observation period.

2.4. Pooled Analysis. A systematic PubMed search was conducted to identify previous studies reporting the cancer risk in patients with AAV diagnosed after 1988. The search was restricted to papers published in English language. Studies who do not report SIR data were excluded. The SIRs were calculated as the sum of the observed cancer cases divided by that of the expected cancer cases in all studies.

2.5. Treatment. Information regarding cumulative CYC doses administered to the patients is not available in the NKBR. In a previous Norwegian study including patients with Wegener's granulomatosis diagnosed between 1988 and 1998, the majority of patients received intravenous CYC with a median cumulative dose of $17 \mathrm{~g}$. In patients receiving oral CYC, the median cumulative dose was $48 \mathrm{~g}$ [22]. Most centers since approximately 2003 have substituted CYC with azathioprine for maintenance treatment, substantially lowering the exposure to CYC [23]. In some patients, rituximab has been used for induction and maintenance treatment. Some patients have also received plasma exchange treatments [24].

2.6. Statistical Analyses. Continuous variables were expressed as medians with 25th and 75th percentiles and 
TABLE 1: Baseline demographics of 419 Norwegian patients with AAV.

\begin{tabular}{|c|c|c|c|c|}
\hline Characteristic & Total & Nonmalignancy & Malignancy & $p$ value \\
\hline Age (median, IQR) & $62(49-72)$ & $61(48-72)$ & $65(56-73)$ & 0.04 \\
\hline Male sex $(\%)$ & $229(55 \%)$ & $200(53 \%)$ & $29(71 \%)$ & 0.03 \\
\hline eGFR (median, IQR) & $23(11-46)$ & $24(11-47)$ & $19(9-39)$ & 0.29 \\
\hline C-ANCA/PR3-ANCA & $237(57 \%)$ & $213(56 \%)$ & $24(59 \%)$ & 0.89 \\
\hline
\end{tabular}

AAV, ANCA-associated vasculitis; IQR, interquartile range; eGFR, estimated glomerular filtration rate, $\mathrm{mL} / \mathrm{min} / 1.73 \mathrm{~m}{ }^{2}$; C-ANCA, cytoplasmic ANCA; PR3ANCA, proteinase 3 ANCA.

categorical variables as numbers (\%). Comparisons of continuous and categorical variables in the baseline characteristics were calculated using the Mann-Whitney $U$ test and the $X^{2}$ or Fisher's exact test, respectively. A two-tailed $p$ value of $\leq 0.05$ and $95 \%$ CI was considered statistically significant. All statistical analyses were performed using the SPSS software, V.23, and STATA software, V.14.

\section{Results}

3.1. Baseline Characteristics. Between 1988 and 2012, 454 patients diagnosed with AAV and glomerulonephritis were identified. Of these, 35 were excluded as a result of cancer diagnosis prior to the observation period. Thus, 419 patients were included in the study cohort. As shown in Table 1, the median age in the cohort was 62 years [interquartile range, 48 to 72 years], and 229 (55\%) were men. The median eGFR at the time of kidney biopsy was $23 \mathrm{~mL} / \mathrm{min} / 1.73 \mathrm{~m}^{2}$ (interquartile range, 11 to 46 ). A positive C-ANCA/PR3-ANCA was found in 237 (57\%) and P-ANCA/MPO-ANCA in 183 (43\%) patients.

The median length of follow-up was 5.7 years (interquartile range, 2.8 to 11.3 ). The mean length of follow-up was 7.2 years (standard deviation, 5.8), and the total number of person-years of observation was 3010. A total of 148 (35\%) patients died during the observation period. Causes of deaths are registered for 138 patients, of which 69 (50\%) died from infectious disease or active inflammation/vasculitis, 36 (26\%) cardiovascular disease, 12 (9\%) malignancy, and 21 (15\%) from other causes. Kidney transplantation was performed in $60(14 \%)$ patients.

3.2. Observed Cancer Cases. During follow-up, 46 cancer cases were reported in $41(9.5 \%)$ patients. The first occurring cancer cases were as follows: NMSC (7 cases), lung (7), prostate (5), hematologic (4), urinary bladder and ureter (3), rectum (3), colon (2), uterus (2), central nerve system (2), unknown primary site (2), thyroid (1), ovary (1), lymphoma (1), and pancreas (1). Five patients had 2 distinct cancer diagnoses; the second occurring cancer cases were as follows: lung (1), NMSC (1), prostate (1), hematologic (1), and stomach (1). Seven patients who received transplants were diagnosed with cancer; the first occurring cancer cases were as follows: lung (4), prostate (1), lymphoma (1), and NMSC (1). Two of the 5 patients with 2 distinct cancers underwent transplant, with the cases of secondary cancer involving the prostate and lungs.
3.3. Comparison of Patients with and without Cancer. The comparison of the patients with and without cancer during follow-up is shown in Table 1. The patients with cancer were significantly older [65 years versus 61 years $(p=0.04)$ ] at the time of AAV diagnosis, and a significantly higher percentage were men $(71 \%$ versus $53 \%(p=0.03))$. There were no significant differences in eGFR and ANCA specificity between those with cancer and those without cancer during follow-up. A higher percentage of patients with cancer died during follow-up [25 (61\%) versus $123(33 \%)(p<0.001)$ ].

3.4. Comparison with the General Population. As shown in Table 2(a), the SIR of overall malignancy was 1.09 (95\% CI 0.81 to 1.49$)$. The SIR of all cancer types, except NMSC, was 0.96 (95\% CI 0.69 to 1.34 ). In the $1-5$-year period after AAV diagnosis, the cancer risk in the study cohort significantly increased compared to that in the general population (SIR, 1.53; 95\% CI 1.01 to 2.32). The SIR did not increase in the first year or $>5$ years of follow-up after AAV diagnosis. In gender, ANCA-, and time period specificity-stratified analyses, SIR of cancer was not significantly increased for males of 1.27 (95\% CI 0.88 to 1.83 ), in the C-ANCA/PR3-ANCA positive group of 1.17 (95\% CI 0.78 to 1.74) and in the 1988-2002 time period of 1.14 (95\% CI 0.78 to 1.68). Compared to the general population, the transplanted patients in the study cohort had a significantly increased malignancy risk (SIR, 2.12; 95\% CI 1.01 to 4.44 ), whereas the nontransplanted patients had no increased risk (SIR, 0.99; 95\% CI 0.71 to 1.39 ).

The SIR calculation for the most common site-specific cancer cases showed significantly increased risks of NMSC (SIR, 3.40; 95\% CI 1.62 to 7.14) and hematologic malignancies (SIR, 3.52; 95\% CI 1.32 to 9.37). The SIR did not significantly increase in any other site-specific cancer type (Table 2(b)).

3.5. Pooled Analysis of the 5 Cohort Studies. The separate and merged SIRs of all cancer types in the present and the 4 previously reported studies are shown in Table 3 and Figure 1. This analysis included a total of 1532 patients, 8801 patientyears of observation, and 236 cancer cases. The merged SIR of all cancer types was 1.72 (95\% CI 1.51 to 1.95$)$ and that of all cancer types, except NMSC, was 1.21 (95\% CI 1.01 to 1.45).

\section{Discussion}

In the present study, there was no statistically significant increase in the cancer incidence in the patients with AAV and glomerulonephritis compared to the age- and sex-matched 
TABLE 2

(a) Standardised incidence ratios for cancers in all sites in the study population

\begin{tabular}{|c|c|c|c|c|}
\hline Characteristic & Observed & Expected & SIR & $95 \% \mathrm{CI}$ \\
\hline All & 41 & 37.5 & 1.09 & 0.81 to 1.49 \\
\hline Non-NMSC & 34 & 35.4 & 0.96 & 0.69 to 1.34 \\
\hline \multicolumn{5}{|l|}{ Sex } \\
\hline Male & 29 & 22.9 & 1.27 & 0.88 to 1.83 \\
\hline Female & 12 & 14.6 & 0.82 & 0.47 to 1.44 \\
\hline \multicolumn{5}{|l|}{ Follow-up period } \\
\hline $0-1$ year & 3 & 4.3 & 0.70 & 0.22 to 2.16 \\
\hline $1-5$ years & 22 & 14.4 & 1.53 & 1.01 to 2.32 \\
\hline $5-10$ years & 11 & 11.1 & 0.99 & 0.55 to 1.78 \\
\hline$>10$ years & 5 & 7.6 & 0.66 & 0.27 to 1.57 \\
\hline \multicolumn{5}{|l|}{ Transplantation } \\
\hline Yes & 7 & 3.3 & 2.12 & 1.01 to 4.44 \\
\hline No & 34 & 34.2 & 0.99 & 0.71 to 1.39 \\
\hline \multicolumn{5}{|l|}{ ANCA serology } \\
\hline C-ANCA/PR3-ANCA & 24 & 20.6 & 1.17 & 0.78 to 1.74 \\
\hline P-ANCA/MPO-ANCA & 17 & 16.9 & 1.01 & 0.63 to 1.62 \\
\hline \multicolumn{5}{|l|}{ Study period } \\
\hline 1988-2002 & 26 & 22.7 & 1.14 & $0.78-1.68$ \\
\hline 2003-2012 & 15 & 14.8 & 1.02 & $0.61-1.68$ \\
\hline
\end{tabular}

SIR, standardised incidence ratio; 95\% CI, 95\% confidence interval; NMSC, nonmelanoma skin cancer; C-ANCA, cytoplasmic ANCA; PR3-ANCA, proteinase 3 ANCA; P-ANCA, perinuclear ANCA; MPO-ANCA, myeloperoxidase ANCA.

(b) Standardised incidence ratios for the most common organ-specific cancers in the study population

\begin{tabular}{lcccc}
\hline Organs & Observed & Expected & SIR & 95\% CI \\
\hline NMSC & 7 & 2.1 & 3.40 & 1.62 to 7.14 \\
Hematologic & 4 & 1.1 & 3.52 & 1.32 to 9.37 \\
Lung & 7 & 4.0 & 1.73 & 0.83 to 3.63 \\
Colon & 2 & 1.2 & 1.73 & 0.43 to 6.93 \\
Urothelium & 3 & 2.0 & 1.48 & 0.47 to 4.59 \\
Prostate & 5 & 7.0 & 0.72 & 0.30 to 1.73 \\
NHL & 1 & 1.2 & 0.86 & 0.12 to 6.12 \\
\hline
\end{tabular}

SIR, standardised incidence ratio; 95\% CI, 95\% confidence interval; NMSC, nonmelanoma skin cancer; NHL, non-Hodgkin lymphoma.

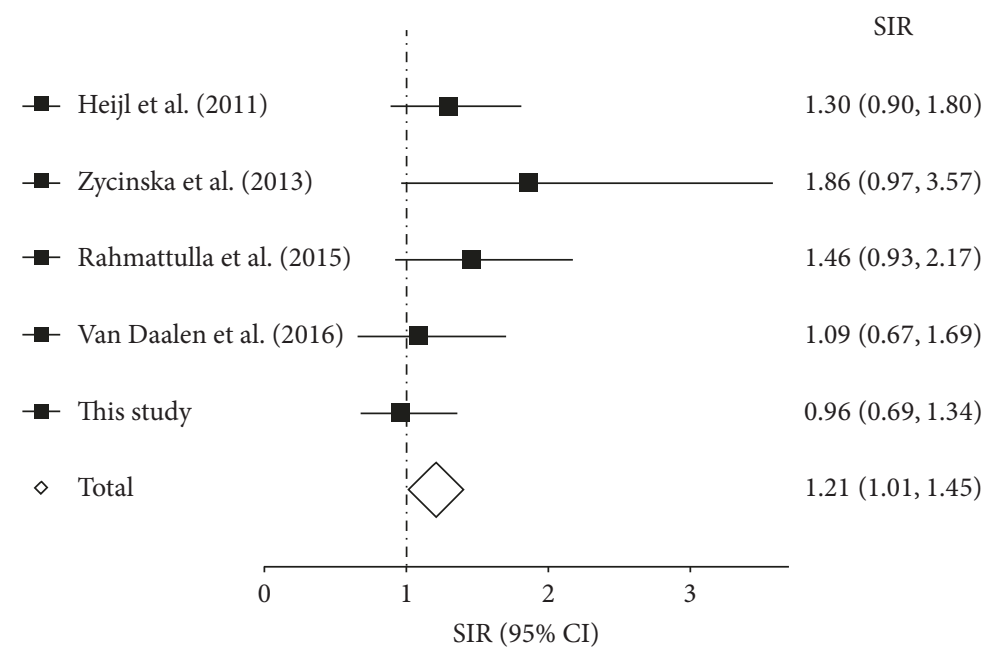

FIGURE 1: Forest plot showing the risk of malignancy except for nonmelanoma skin cancer in observational studies of patients with ANCAassociated vasculitis. SIR, standardised incidence ratio; 95\% CI, 95\% confidence interval. 


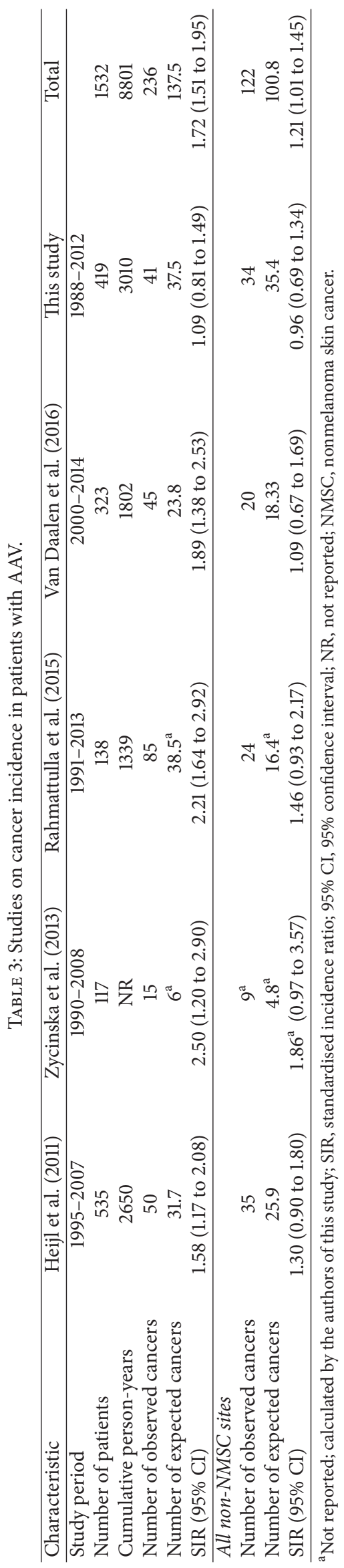


general population (SIR, 1.09; 95\% CI 0.89 to 1.49). Excluding the study by Holle et al. that reported an SIR of 0.82 (95\% CI 0.45 to 1.38) [25], most previous studies, including those investigating patients with AAV diagnosed after 1988, have reported a significantly increased cancer risk $[3-5,9,11,13,14$, 19]. A couple of methodological discrepancies might partially explain the contrasting findings between the present and the majority of previous studies. First, basal skin cell carcinomas are not registered in the Norwegian Cancer Registry and were thus excluded in our analysis. Second, among patients with diagnoses of several cancers, we only included the primary cancer when calculating the SIRs. In contrast, other studies have included subsequent cancer cases, particularly NMSC, in their SIR estimates.

The studies investigating cancer incidences in patients with AAV diagnosed after 1988 reflect contemporary treatment regimens, in which NMSC accounts for the majority of the observed increased malignancy risk $[5,11,13,19]$. In the present study, the SIR decreased from 1.09 to 0.96 (95\% CI 0.69 to 1.34 ) when NMSC cases were excluded. Further, in the pooled analysis (Table 3), the SIR decreased from 1.72 (95\% CI 1.51 to 1.95 ) to 1.21 (95\% CI 1.01 to 1.45 ) after excluding NMSC. Moreover, part of the residual cancer risk after excluding NMSC can be attributed to posttransplant malignancies, with the SIR posttransplant of 2.12 (95\% CI 1.01 to 4.44 ) in the present study and 4.31 (95\% CI 1.17 to 11.04) in that by Van Daalen et al. [19].

In the organ-specific subanalysis, the SIR of NMSC was 3.40 (95\% CI 1.62 to 7.14 ), which is consistent with that of previous studies $[5,9,11,13,14,19]$. An increased risk of NMSC is observed in immunocompromised patients and associated with both environmental factors as chronic human papillomavirus infection [26-29] and a direct effect of individual immunosuppressant, for example, azathioprine $[19,30]$. A significantly increased hematologic cancer risk was also found (SIR, 3.52; 95\% CI 1.32 to 9.37). Traditionally, CYC use was associated with a very high risk of acute myelogenous leukemia $[9,14,31]$. Interestingly, no case of this cancer form was observed in our cohort, and the increased hematologic malignancy risk was caused by 2 cases of myelodysplastic syndrome and 2 cases of chronic lymphocytic leukemia. To what extent these cases are related to CYC-based therapy or immunosuppression specifically is unclear [32]. In comparison, Zycinska et al. found an increased risk of acute myelogenous leukemia in their study (SIR, 4.3; 95\% CI 2.1 to 11.7). Notably, oral CYC was used in the majority of their patients, and $22 \%$ received $>36 \mathrm{~g}$ of cumulative CYC dosage. In addition, a significantly increased urothelial cancer risk was also found in their study, in contrast to those of other recent groups (SIR, 3.4; 95\% CI 1.6 to 5.2) [11].

Some previous groups have indicated that the cancer risk, perhaps caused by a higher tendency of relapses and thus higher cumulative immunosuppressive drug doses, is higher in C-ANCA/PR3-ANCA than in P-ANCA/MPOANCA positive patients $[5,13]$. In contrast, we observed no significantly increased risk in these subgroups compared to the general population.

An important measure to reduce cancer risk in patients with AAV has been replacing CYC with azathioprine for maintenance treatment. This practice change occurred around 2003 concurrent with the publication of the CYCAZAREM study $[23,33]$. In the present study cohort, SIR of cancer was not significantly increased, neither in the 1988-2002 nor in the 2003-2012 time periods. Of notice, in a study including a subgroup of the 1988-2002 cohort, cumulative doses of CYC were found fairly low, median 17 grams in intravenous CYC-treated patients [34].

The findings by Van Daalen et al. indicate that rituximab use is associated with a substantially lower risk of malignancy. However, this difference was primarily related to excess NMSC cases. The calculated SIR of cancer, except NMSC, was 1.14 (95\% CI 0.49 to 2.25 ) with CYC and 0.88 (95\% CI 0.11 to 3.19) with rituximab treatment. Thus, the SIR of cancer, except NMSC, was only 1.30 -fold higher (CI not reported by Van Daalen et al.) in the CYC group than in the rituximab group. Further, the SIR of cancer, except NMSC, was only marginally higher in the present CYC-treated study cohort than in the rituximab group in their study, estimated SIR of 0.96 (95\% CI 0.69 to 1.34 ) and 0.88 (95\% CI 0.11 to 3.19 ), respectively. Finally, no adjustment for posttransplantation malignancies has been performed when comparing CYC- and rituximabtreated patients [19]. In summary, whether replacing lowdose CYC regimens with rituximab would have a beneficial effect on non-NMSC malignancy risk remains uncertain.

NMSC occurrence is still substantially increased in patients with AAV. Although NMSC by no means should be considered as an inconsequential morbidity, deaths caused by these tumors are rare [35]. A number of measures can be taken to reduce the risk and morbidity related to NMSC in immunosuppressed patients; the most important measures include limiting sun exposure of the skin and vigilant monitoring with early NMSC detection and treatment when they appear. Interestingly, Van Daalen et al. observed that rituximab use is associated with a lower NMSC risk than CYC use, which represents a new possible solution to such complication in patients with AAV [19]. However, the decision to replace CYC with rituximab as the first-line treatment in patients with AAV must also include considerations, such as efficacy, treatment-related complications, and cost-benefit.

The major strengths of the present study include its population-based approach and identification of patients with AAV from quality registries with histologic and serologic data. Information on expected and observed cancer cases was retrieved from the same registry, the Norwegian Cancer Registry, which limits potential information biases in the SIR calculation. This registry also has high accuracy and only few missing cases owing to mandatory reporting of cancer cases. Moreover, the pooled analysis strengthens the statistical power and increases the detection rate of significant differences. Some weaknesses of our study must be admitted. Most importantly, we could not correlate our findings to the cumulative CYC doses administered. Treatment data were unavailable in the NKBR. However, we have shown from previous reports of this cohort that these patients have received therapy according to international recommendations [2224]. Another weakness is the lack of information regarding extrarenal and relapsing disease. Relapsing disease in particular is associated with increased treatment length and 
cumulative doses of immunosuppressive drugs that might affect risk of cancer development. Also, owing to a lack of sufficient data, we could not calculate pooled SIRs for singlesite cancers or for malignancy after excluding posttransplant cancers.

In summary, we have demonstrated that the risk of malignancy in patients with AAV and glomerulonephritis is not significantly increased. However, significantly increased NMSC, hematologic malignancy, and posttransplantation cancer risks were found. These findings indicate the presence of associations, although relatively weak, between immunosuppression and cancer development. However, recently published data suggest that substituting CYC with rituximab could eliminate the risk of developing NMSC. Our findings confirm that the long-term international efforts of developing CYC-minimizing strategies had an important beneficial effect on cancer morbidity in patients with AAV.

\section{Conflicts of Interest}

The authors declare that there are no conflicts of interest regarding the publication of this article.

\section{Authors' Contributions}

All authors contributed to the generation of hypotheses. Tor Åge Myklebust and Bjørn Møller performed statistical analyses; Sanjeevan Sriskandarajah and Rune Bjørneklett drafted the manuscript. All of the authors contributed to revisions and approved the final version of the manuscript.

\section{Acknowledgments}

The authors thank all nephrologists and pathologists in Norway who reported important data to the Norwegian Kidney Biopsy Registry. They also thank Torbjørn Leivestad for data linkage of the study cohort with the Norwegian Renal Registry.

\section{References}

[1] J. C. Jennette and R. J. Falk, "Small-vessel vasculitis," The New England Journal of Medicine, vol. 337, no. 21, pp. 1512-1523, 1997.

[2] A. S. Fauci, B. F. Haynes, P. Katz, and S. M. Wolff, "Wegener's granulomatosis: prospective clinical and therapeutic experience with 85 patients for 21 years," Annals of Internal Medicine, vol. 98, no. 1, pp. 76-85, 1983.

[3] G. S. Hoffman, G. S. Kerr, R. Y. Leavitt et al., "Wegener granulomatosis: an analysis of 158 patients," Annals of Internal Medicine, vol. 116, no. 6, pp. 488-498, 1992.

[4] K. W. A. Westman, P. G. Bygren, H. Olsson, J. Ranstam, and J. Wieslander, "Relapse rate, renal survival, and cancer morbidity in patients with Wegener's granulomatosis or microscopic polyangiitis with renal involvement," Journal of the American Society of Nephrology, vol. 9, no. 5, pp. 842-852, 1998.

[5] C. Heijl, L. Harper, O. Flossmann et al., "Incidence of malignancy in patients treated for antineutrophil cytoplasm antibody-associated vasculitis: Follow-up data from European Vasculitis Study Group clinical trials," Annals of the Rheumatic Diseases, vol. 70, no. 8, pp. 1415-1421, 2011.
[6] C. Talar-Williams, Y. M. Hijazi, M. McClellan et al., "Cyclophosphamide-induced cystitis and bladder cancer in patients with Wegener granulomatosis," Annals of Internal Medicine, vol. 124, no. 5, pp. 477-484, 1996.

[7] J. Robson, H. Doll, R. Suppiah et al., "Damage in the ANCAassociated vasculitides: Long-term data from the European Vasculitis Study Group (EUVAS) therapeutic trials," Annals of the Rheumatic Diseases, vol. 74, no. 1, pp. 177-184, 2015.

[8] C. Mukhtyar, "SP0095 EULAR/ERA-EDTA Recommendations for The Management of ANCA-Associated Vasculitis," Annals of the Rheumatic Diseases, vol. 75, no. Suppl 2, pp. 24.1-24, 2016.

[9] M. Faurschou, I. J. Sorensen, L. Mellemkjaer et al., "Malignancies in wegener's granulomatosis: incidence and relation to cyclophosphamide therapy in a cohort of 293 patients," The Journal of Rheumatology, vol. 35, no. 1, pp. 100-105, 2008.

[10] M. Faurschou, L. Mellemkjaer, A. Voss, K. K. Keller, I. T. Hansen, and B. Baslund, "Prolonged risk of specific malignancies following cyclophosphamide therapy among patients with granulomatosis with polyangiitis," Rheumatology, vol. 54, no. 8, pp. 1345-1350, 2015.

[11] K. Zycinska, J. Kostrzewa-Janicka, A. Nitsch-Osuch, and K. Wardyn, "Cancer Incidence in Pulmonary Vasculitis," in Neurobiology of Respiration, vol. 788 of Advances in Experimental Medicine and Biology, pp. 349-353, Springer, Dordrecht, Netherlands, 2013.

[12] A. Knight, J. Askling, F. Granath, P. Sparen, and A. Ekbom, "Urinary bladder cancer in Wegener's granulomatosis: risks and relation to cyclophosphamide," Annals of the Rheumatic Diseases, vol. 63, no. 10, pp. 1307-1311, 2004.

[13] C. Rahmattulla, A. E. Berden, S.-C. Wakker et al., "Incidence of malignancies in patients with antineutrophil cytoplasmic antibody-associated vasculitis diagnosed between 1991 and 2013," Arthritis \& Rheumatology, vol. 67, no. 12, pp. 3270-3278, 2015.

[14] A. Knight, J. Askling, and A. Ekbom, "Cancer incidence in a population-based cohort of patients with Wegener's granulomatosis," International Journal of Cancer, vol. 100, no. 1, pp. 82$85,2002$.

[15] M. Walsh, M. Faurschou, A. Berden et al., "Long-term followup of cyclophosphamide compared with azathioprine for initial maintenance therapy in anca-associated vasculitis," Clinical Journal of the American Society of Nephrology, vol. 9, no. 9, pp. 1571-1576, 2014.

[16] R. M. Smith, "Update on the treatment of ANCA associated vasculitis," La Presse Médicale, vol. 44, no. 6, pp. e241-e249, 2015.

[17] R. L. Rhee, S. L. Hogan, C. J. Poulton et al., "Trends in LongTerm Outcomes Among Patients With Antineutrophil Cytoplasmic Antibody-Associated Vasculitis With Renal Disease," Arthritis \& Rheumatology, vol. 68, no. 7, pp. 1711-1720, 2016.

[18] K. De Groot, L. Harper, D. R. W. Jayne et al., "Pulse versus daily oral cyclophosphamide for induction of remission in antineutrophil cytoplasmic antibody-associated vasculitis: a randomized trial," Annals of Internal Medicine, vol. 150, no. 10, pp. 670-680, 2009.

[19] E. E. Van Daalen, R. Rizzo, A. Kronbichler et al., "Effect of rituximab on malignancy risk in patients with ANCA-Associated vasculitis," Annals of the Rheumatic Diseases, vol. 76, no. 6, pp. 1064-1069, 2016.

[20] I. K. Larsen, M. Småstuen, T. B. Johannesen et al., "Data quality at the Cancer Registry of Norway: An overview of comparability, completeness, validity and timeliness," European Journal of Cancer, vol. 45, no. 7, pp. 1218-1231, 2009. 
[21] A. S. Levey, J. Coresh, T. Greene et al., "Expressing the modification of diet in renal disease study equation for estimating glomerular filtration rate with standardized serum creatinine values," Clinical Chemistry, vol. 53, no. 4, pp. 766-772, 2007.

[22] K. Aasarod, "Renal histopathology and clinical course in 94 patients with Wegener's granulomatosis," Nephrology Dialysis Transplantation, vol. 16, no. 5, pp. 953-960.

[23] S. Sriskandarajah, K. Aasarød, S. Skrede, T. Knoop, A. V. Reisæter, and R. Bjørneklett, "Improved prognosis in Norwegian patients with glomerulonephritis associated with anti-neutrophil cytoplasmic antibodies," Nephrology Dialysis Transplantation, vol. 30, pp. i67-i75, 2015.

[24] E. Besada, W. Koldingsnes, and J. C. Nossent, "Long-term efficacy and safety of pre-emptive maintenance therapy with rituximab in granulomatosis with polyangiitis: results from a single centre," Rheumatology, vol. 52, no. 11, Article ID ket257, pp. 2041-2047, 2013.

[25] J. U. Holle, W. L. Gross, U. Latza et al., "Improved outcome in 445 patients with Wegener's granulomatosis in a German vasculitis center over four decades," Arthritis \& Rheumatology, vol. 63, no. 1, pp. 257-266, 2011.

[26] N. Reusser, C. Downing, J. Guidry, and S. Tyring, "HPV Carcinomas in Immunocompromised Patients," Journal of Clinical Medicine, vol. 4, no. 2, pp. 260-281, 2015.

[27] A. E. Grulich, M. T. van Leeuwen, M. O. Falster, and C. M. Vajdic, "Incidence of cancers in people with HIV/AIDS compared with immunosuppressed transplant recipients: a metaanalysis," The Lancet, vol. 370, no. 9581, pp. 59-67, 2007.

[28] E. N. Pritchett, A. Doyle, C. M. Shaver et al., "Nonmelanoma skin cancer in nonwhite organ transplant recipients," JAMA Dermatology, vol. 152, no. 12, pp. 1348-1353, 2016.

[29] S. Euvrard, J. Kanitakis, and A. Claudy, "Skin cancers after organ transplantation," The New England Journal of Medicine, vol. 348, no. 17, pp. 1681-1691, 2003.

[30] P. O’Donovan, C. M. Perrett, and X. Zhang, "Azathioprine and UVA light generate mutagenic oxidative DNA damage," Science, vol. 309, no. 5742, pp. 1871-1874, 2005.

[31] J. Pedersen-Bjergaard, S. Olesen Larsen, J. Struck et al., "Risk of therapy-related leukaemia and preleukaemia after Hodgkin's disease. Relation to age, cumulative dose of alkylating agents, and time from chemotherapy," The Lancet, vol. 330, no. 8550, pp. 83-88, 1987.

[32] V. Bîrluţiu, E. C. Rezi, R. M. Bîrluţiu, and I. S. Zaharie, "A rare association of chronic lymphocytic leukemia with c-ANCApositive Wegener's granulomatosis: A case report," World Journal of Surgical Oncology, vol. 14, no. 1, article no. 145, 2016.

[33] D. Jayne, N. Rasmussen, K. Andrassy et al., "A randomized trial of maintenance therapy for vasculitis associated with antineutrophil cytoplasmic autoantibodies," The New England Journal of Medicine, vol. 349, no. 1, pp. 36-44, 2003.

[34] K. Aasarod, B. M. Iversen, J. Hammerstrom, L. Bostad, L. Vatten, and S. Jorstad, "Wegener's granulomatosis: clinical course in 108 patients with renal involvement," Nephrology Dialysis Transplantation, vol. 15, no. 5, pp. 611-618, 2000.

[35] C. Newlands, R. Currie, A. Memon, S. Whitaker, and T. Woolford, "Non-melanoma skin cancer: United Kingdom National Multidisciplinary Guidelines," The Journal of Laryngology \& Otology, vol. 130, no. S2, pp. S125-S132, 2016. 


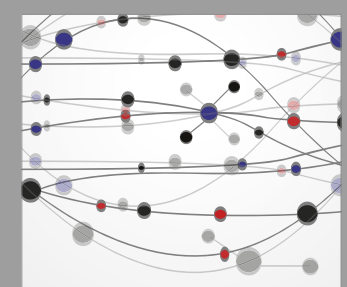

The Scientific World Journal
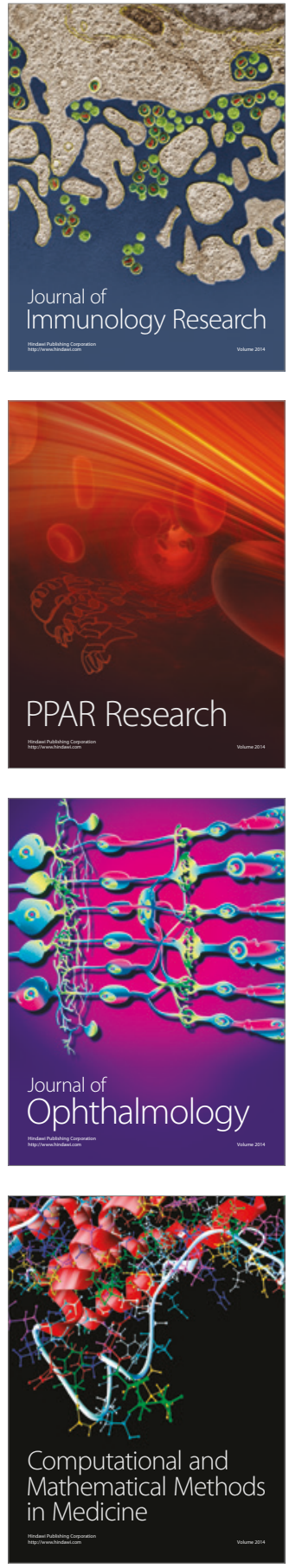

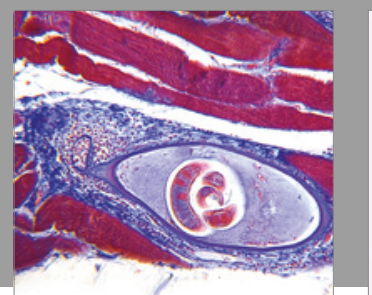

Gastroenterology Research and Practice
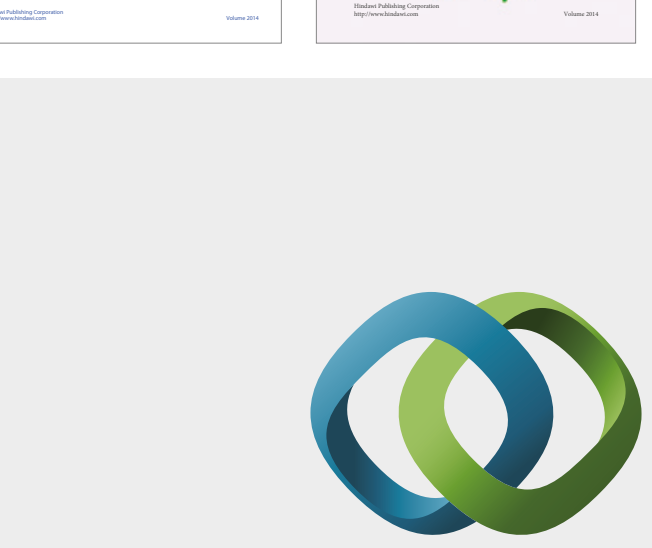

\section{Hindawi}

Submit your manuscripts at

https://www.hindawi.com
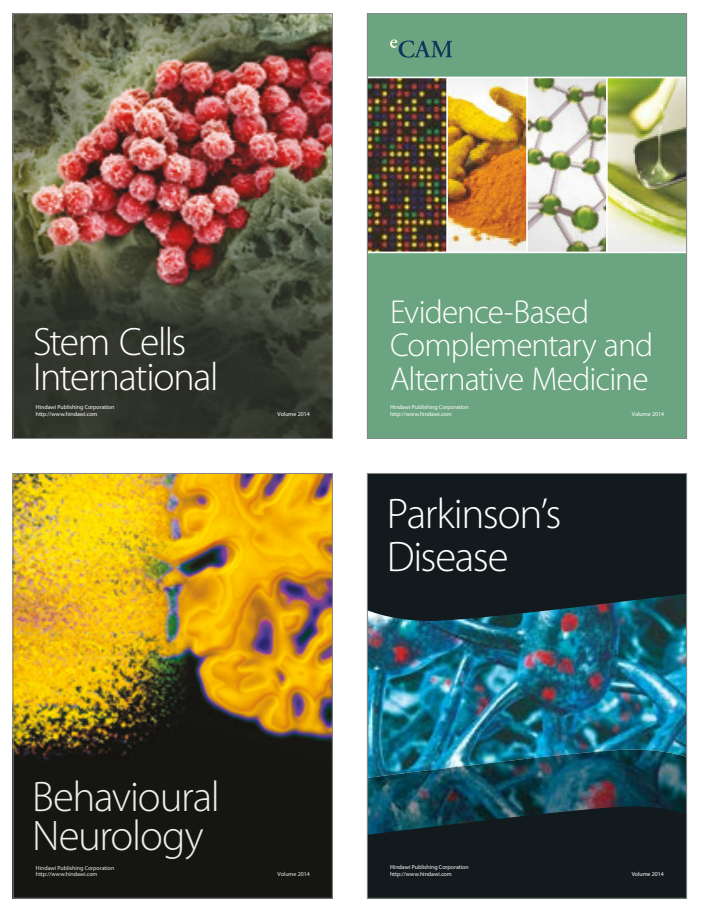
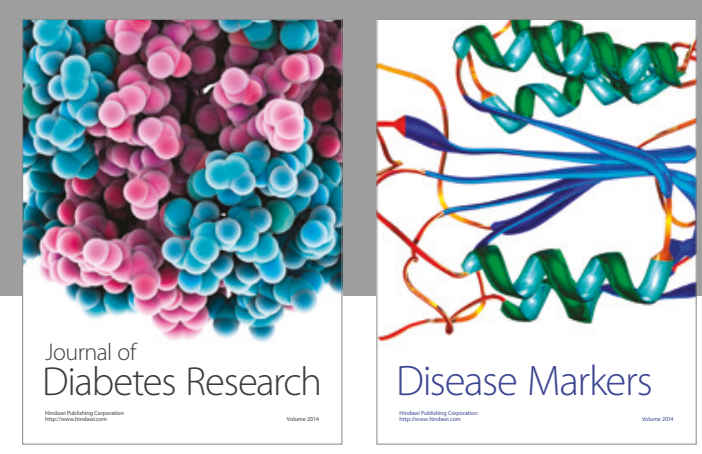

Disease Markers
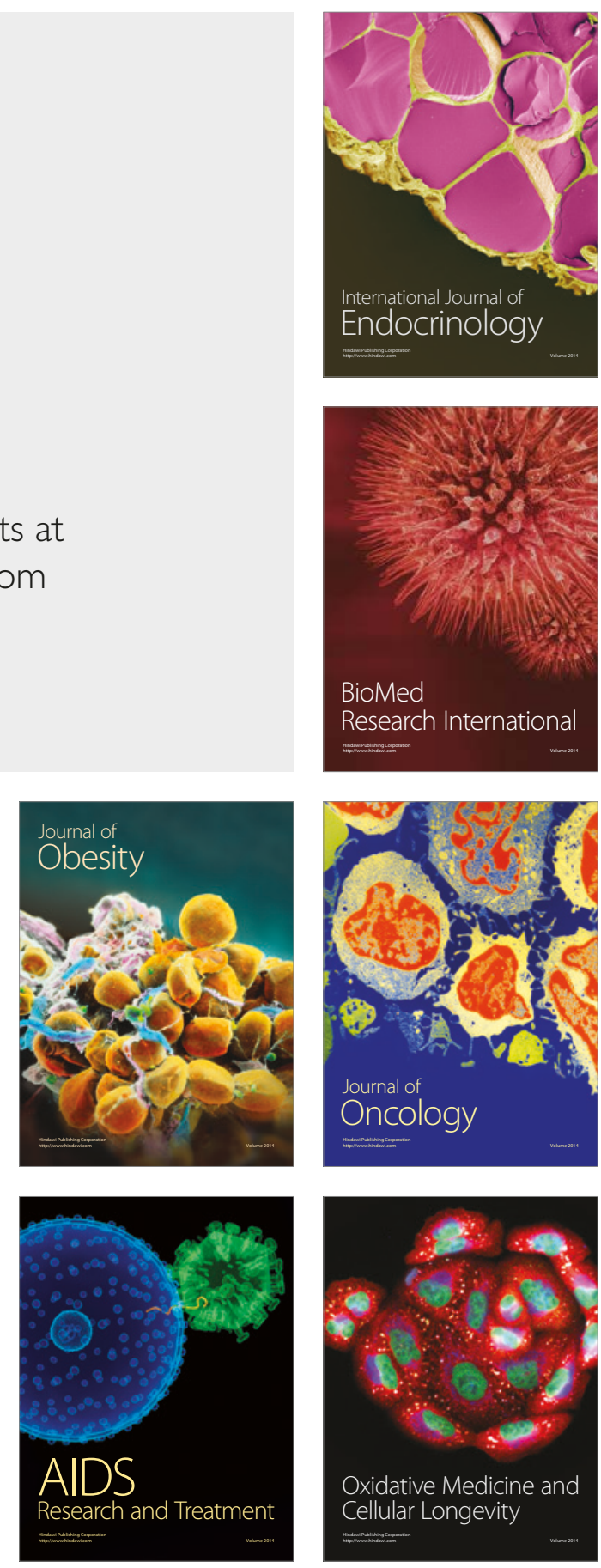